\title{
O DISCURSO DE SUSTENTABILIDADE E A PESQUISA ACADEMICA SOBRE RELATO INTEGRADO (IR) NO SETOR PUBLICO
}

Franciele Machado de Souza'

Luiz Panhoca²

\section{Resumo:}

Objetivo:Com base na teoria do discurso de Laclau e Mouffe e na expectativa de ampliar o entendimento da sustentabilidade corporativa para que as vozes acadêmicas possam oferecer uma crítica contínua e novas alternativas para este discurso, estabeleceu-se como objetivo analisar a construção do discurso de sustentabilidade nos resultados de pesquisas sobre os Relatos Integrados (IRs) de empresas do setor público.

Método: As pesquisas publicadas, que analisaram os IRs destas organizações, foram examinadas para considerar como o discurso hegemônico estava construído nos 18 artigos científicos, selecionados/ identificados na base de dados Scopus com os termos "integrated reporting", "state-owned" e "public sector". Os estudos foram classificados quanto as suas metodologias e a forma de apropriação do discurso em torno do ponto nodal sustentabilidade.

Resultados: Os resultados revelaram que o discurso de sustentabilidade presente nas pesquisas revisadas estava relacionado ao desenvolvimento econômico, à estratégia e ao "business-case". No entanto, existem evidências a adoção ao IR tem o potencial de impulsionar a mudança organizacional para integrar a sustentabilidade à atividade principal da organização. Em suma, os achados sugerem que a adoção ao IR, por empresas do setor público, não modificou o discurso hegemônico de sustentabilidade financeira.

Contribuições: Assim, o artigo contribui com a literatura de IR ao revelar a necessidade das pesquisas sobre sustentabilidade ampliarem a investigação nos relatórios, a partir de novos enfoques, análises críticas e pesquisas normativas que objetivem propor adaptações ao IR nas instituições públicas, e até propor outros frameworks.

Palavras-chave: Discurso; Relato Integrado; Sustentabilidade; Setor público; Hegemonia. 


\title{
SUSTAINABILITY DISCOURSE AND ACADEMIC RESEARCH ON INTEGRATED REPORTING (IR) IN THE PUBLIC SECTOR
}

\begin{abstract}
:
Objective: Based on the theory of discourse by Laclau and Mouffe and on the expectation of broadening the understanding of corporate sustainability so that academic voices can offer continuous criticism and new alternatives for this discourse, the objective was to analyze the construction of the discourse of sustainability in the results of research on the Integrated Reports (IRs) of public sector companies. Method: The published research, which analyzed the IRs of these organizations, was examined to consider how the hegemonic discourse was built on the 18 scientific articles, selected/identified in the Scopus database with the terms "integrated reporting", "state-owned" and "Public sector". The studies were classified according to their methodologies and the form of speech appropriation around the nodal point of sustainability.

Results: The results revealed that the sustainability discourse present in the reviewed surveys was related to economic development, strategy and the "business-case". However, there are evidences that adoption of IR has the potential to drive organizational change to integrate sustainability into the organization's core business. In short, the findings suggest that the adoption of IR by public sector companies has not changed the hegemonic discourse of financial sustainability.

Contributions: Thus, the article contributes to the IR literature by revealing the need for research on sustainability to expand research in reports, from new approaches, critical analyzes and normative research that aims at proposing adaptations to IR in public institutions, and even proposing other frameworks.
\end{abstract}

Keywords: Discourse; Integrated Reporting; Sustainability; Public Sector; Hegemony. 


\section{INTRODUÇÃO}

A preocupação com o desenvolvimento sustentável, bem como a noção complexa pela qual as questões sociais e ambientais devem ser tratadas, seja a nível político, pessoal ou organizacional, tem sido crescente na literatura contábil (Gray, 2010). No entanto, o conceito de sustentabilidade e, os seus determinantes sociais, ambientais e econômicos têm sido frequentemente interpretados de diferentes maneiras (Drexhage \& Murphy, 2010) e geralmente analisados de forma isolada (Bebbington \& Gray, 2001; Gray, 2010).

Para Gray (2010) sustentabilidade e desenvolvimento sustentável não são conceitos "estáticos". Embora "sustentar" tenha uma miríade de significados, a lógica capitalista foi rápida na tentativa de limitar o escopo de significação, limitando a definição como uma forma de crescimento econômico (Voss, Carter \& Salotti, 2017).

Essa concepção surge em razão do processo de degradação econômica, fragilidade política e destruição da natureza como tentativa de amenizar a gérmen do sistema de produção capitalista: exploração, destruição e alienação (Vizeu, Meneghetti \& Seifert, 2012).

Nos últimos 20 anos, o setor público registrou mudanças sociais, políticas e econômicas que levaram à evolução do conceito de prestação de contas (Veltri \& Silvestri, 2015). As organizações públicas também adotaram práticas de sustentabilidade, mas com sinais mais lentos (Figueira et al., 2018). Estas instituições prestam serviços que afetam a vida das pessoas e devem apresentar comportamentos responsáveis com a finalidade de salvaguardar os estilos de vida sustentáveis das gerações futuras (Dumay, Guthrie \& Farneti, 2010).

As múltiplas interpretações da sustentabilidade e a necessidade de regulá-la por meio de diferentes frameworks e iniciativas (tais como: Global Reporting Initiative - GRI, Sustainability Accounting Standards Board - SASB), deram origem a novas interpretações ontológicas e epistemológicas do conceito, permitindo uma acomodação das ações por parte das empresas (Gray, 2010). Esses frameworks variam em termos de escopo, especificação, questões abordadas e metodologia (Busco, Giovannoni, Graná \& Izzo, 2018).

A natureza multidimensional dos relatórios de sustentabilidade e a necessidade de integrar o desempenho financeiro e não financeiro no mesmo relatório foram enfatizadas pelo International Integrated Reporting Council (IIRC) em seu Framework Internacional do Relato Integrados (IIRF), emitido em 2013 (Busco et al., 2018). O objetivo do Relato Integrado (IR) é fornecer informações sobre desempenho financeiro e não financeiro e sua influência no processo de criação de valor das organizações em um único documento (International Integrated Reporting Council - IIRC, 2013).

Embora o IR tenha como objetivo mobilizar as informaçōes corporativas, facilitando o envolvimento e o ativismo dos stakeholders da organização, críticas surgiram ao IR (Busco, Frigo, Paolo \& Angelo, 2013; Milne \& Gray, 2013; Brown \& Dillard, 2014; Flower, 2015; Thompson, 2015; Humphrey, O'Dwyer \& Unerman, 2017) e outros revelam o papel "aspiracional" da adoção do IR (Gibassier, Rodrigue \& Arjaliès, 2018; Busco et al., 2018).

Para Humphrey et al. (2017), há pouca indicação nos materiais fornecidos pelo IIRC sobre como e por que o IR atende às necessidades da sustentabilidade. Brown e Dillard (2014) criticam o IIRF, pois, defende as lentes da maximização da riqueza dos acionistas, enfatizando as oportunidades de ganho mútuo ao aproximar a sustentabilidade nas organizações. Além disso, Flower (2015) critica a maneira 
pela qual os objetivos originais do IIRC, em apoiar o desenvolvimento sustentável, foram abandonados e substituídos pelo conceito de criação de valor na versão final do framework.

Busco et al. (2018) relatam que, em vez de preencher a sustentabilidade com significado estável, os indivíduos são atraídos pelas lacunas deixadas pelas representações contábeis, levando ao desenvolvimento de novas práticas e ao desdobramento de significados em espaços discursivos. Neste sentido, Gibassier et al. (2018) enfatizam que se as organizações desejam realizar todo o potencial do IR, elas precisam garantir que suas características aspiracionais estejam alinhadas com a visão gerencial da empresa.

Apesar de uma quantidade crescente de pesquisas sobre a sustentabilidade nos IRs, o setor público foi deixado de fora do escopo desses estudos (Dumay, Bernardi, Guthrie \& Demartini, 2016; Figueira et al., 2018). Surgem questionamentos concernentes a como os discursos sobre sustentabilidade se tornaram significativos por meio da adoção do IR e as contradições inerentes entre o crescimento econômico, desenvolvimento sustentável e os limites ecológicos nestas organizações. Nesse contexto, buscou-se discutir a seguinte questão de pesquisa: Como se constrói o discurso de sustentabilidade nos Relatos Integrados de empresas do setor público? Assim, este estudo teve o objetivo de analisar a construção do discurso de sustentabilidade nos resultados de pesquisas sobre os IRs de empresas do setor público.

Em especial, aceito o apelo de Higgins et al. (2014), Tregidga et al. (2014), Tregidga et al. (2018) e Busco et al. (2018) por mais estudos críticos do discurso da sustentabilidade, e até que ponto a retórica e os significantes vazios de sustentabilidade, oferece uma oportunidade para mudanças reais nas atividades organizacionais. Além disso, as pesquisas sobre o IR no setor público estão em fase inicial (Dumay et al., 2016; Guthrie, Manes-Rossi \& Orelli, 2017) e não foram encontradas pesquisas brasileiras com a temática.

O método de pesquisa consistiu em uma revisão da literatura combinada com teoria do discurso de Laclau e Mouffe (1985). A pesquisa foi delimitada aos artigos publicados em periódicos internacionais, porque não se identificou literatura sobre este tema nos nacionais.

Os resultados demonstram que, no discurso corporativo em torno do ponto nodal sustentabilidade, nos estudos revisados, são feitas referências a sustentar a economia, o meio ambiente e a sociedade. No entanto, não está claro que a adoção do IR mudou o discurso hegemônico de sustentabilidade financeira nos artigos revisados. As principais lacunas permanecem, uma vez que os estudos estão em sua fase inicial.

\section{REFERENCIAL TEÓRICO}

\subsection{Sustentabilidade: uma perspectiva discursiva aplicada ao setor público}

Embora tenha uma história muito mais longa, o desenvolvimento sustentável ganhou atenção por meio do Relatório Brundtland (World Commission on Environment and Development - WCED, 1987) e uma questão importante na agenda corporativa (Tregidga et al., 2018). O relatório foi amplamente reconhecido por profissionais e acadêmicos como o primeiro documento oficial que popularizou o conceito de desenvolvimento sustentável e posicionou-o como um tópico de importância nacional e global, com foco na reconciliação das necessidades das gerações presentes e futuras (Granà, 2018). O Relatório Brundtland definiu desenvolvimento sustentável como "desenvolvimento que atenda às necessidades do presente sem comprometer a capacidade das gerações futuras de atender às suas próprias necessidades" (WCED, 1987, p. 43).

Este conceito apresenta dois aspectos principais: necessidades e limites (WCED, 1987). O conceito de necessidades, em particular, as necessidades essenciais dos pobres do mundo, às quais deve ser dada prioridade; e a ideia de limitações impostas pelo estado da tecnologia e organização social à capacidade do ambiente de atender às necessidades presentes e futuras (WCED, 1987). Apesar deste esforço signifi- 
cativo para debater como os problemas ambientais, o desenvolvimento social e a viabilidade econômica de longo prazo estão ligados ao conceito de sustentabilidade, porém parece que ainda não há consenso sobre o que realmente significa sustentabilidade (Granà, 2018).

A construção dominante de sustentabilidade foi proposta por Elkington (1997) em seu livro Cannibals with forks: The triple bottom line of 21 st century business, com o objetivo de equilibrar questões econômicas, sociais e ambientais nos relatórios organizacionais (Granà, 2018). A abordagem do Triple Bottom Line (TBL) visava a orientar as organizações para alcançar um equilíbrio entre questões econômicas, ambientais e sociais (Elkington, 1997). OTBL procura explicar a lucratividade financeira juntamente com os impactos sociais e ecológicos de uma organização (Zappettini \& Unerman, 2016). Assim, por meio da TBL, as organizações são incentivadas a adicionar duas linhas de fundo, além da medida comum do lucro corporativo para medir seu impacto social e ambiental (Granà, 2018). Contudo, a ambiguidade permaneceu, pois, como cada uma das três dimensões e as interações entre elas são entendidas, tem-se conceituações muito diferentes (Tregidga et al., 2018).

A heurística do TBL de Elkington (1997) baseou-se, também, na possibilidade de uma ação comercial responsável ser boa para a sociedade, boa para os negócios e para o meio ambiente (Milne, Kearins \& Walton, 2006). Para Gray (2010), existe uma infinidade de potenciais estados de sustentabilidade que podem parecer um exercício de imaginação, mas uma imaginação baseada em um conjunto de decisões sobre: o que deve ser sustentado; por quanto tempo; e em que condição.

Os rótulos de sustentabilidade fraco e forte podem ser vistos como duas extremidades opostas de um continuum, sendo o equilíbrio dos trade-offs que definiu a diferença entre as conceituações (Tregidga, 2007). No entanto, essas representações têm sido criticadas por considerar a sustentabilidade como compartimentalizada, sem destacar como as dimensões ambiental, social e econômica se afetam, limitando o conceito a um momento e ignorando sua dinâmica ao longo do tempo (Stiglitz \& Fitoussi, 2009). Para Gray (2010) a interação entre a infinidade de estados potenciais da sustentabilidade e a gama de estratégias epistemológicas que podem ser adotadas, tem como consequencia que não há claramente nenhuma "sustentabilidade" que possa ser conhecida e contabilizada.

O setor público desempenha um papel significativo na garantia da sustentabilidade ambiental e da responsabilidade, e está cada vez mais sendo pressionado a rever seu conceito de sustentabilidade (Silva, Yapa \& Vesty, 2020). Assim, deve exibir um maior grau de sustentabilidade, maior resposta às petições e expectativas dos stakeholders, compromisso público mais forte e um maior grau de consulta nos processos de tomada de decisão (García -Sánchez et al., 2013). No entanto, Figueira et al. (2018) revelam que as instituições públicas demonstram um baixo nível de adoção a políticas e práticas integradas de sustentabilidade, apesar de esperadas tendências positivas relacionadas às práticas sociais e econômicas.

\subsection{Framework do Relato Integrado (IR)}

O IIRC foi fundado por iniciativa de duas organizações líderes no campo da contabilidade para a sustentabilidade:The Prince's Accounting for Sustainability Project (A4S) e a Global Reporting Initiative (GRI). A criação do IIRC está relacionada ao discurso proferido pelo Príncipe de Gales, em dezembro de 2009, no qual pedia a criação desse órgão (Flower, 2015). O IIRC é formado por uma coalizão global de reguladores, investidores, empresas, standard setters, profissionais contábeis e organizações não governamentais (ONGs) (IIRC, 2013). 
Para o IIRC, o pensamento integrado e o IR atuaram como uma força de estabilidade financeira e sustentabilidade (IIRC, 2013). Embora o IR teve como objetivo principal informar aos provedores de capital financeiro como uma organização cria valor ao longo do tempo, ele pode beneficiar os demais stakeholders (tais como, funcionários, clientes, fornecedores, parceiros comerciais, comunidades locais, legisladores, reguladores e formuladores de políticas) (IIRC, 2013).

Os IRs reúnem informações materiais sobre a estratégia, governança, desempenho e perspectivas de uma organização, de maneira a refletir o contexto comercial, social e ambiental em que opera (Flower, 2015). As organizações podem reportar seu uso ou impacto em seis categorias de capitais: financeiro, manufaturado, intelectual, humano, social e de relacionamento e natural. Assim, o IR visa a destacar os trade-offs entre os seis capitais e mostrar como os negócios criam valor ao longo do tempo. O objetivo do IR é identificar riscos, oportunidades e resultados que afetam a capacidade da organização de criar valor (IIRC, 2013). Além disso, a sustentabilidade do empreendimento deve ser prevista, destacando os planos para manter e aprimorar os seis principais capitais que contribuem na criação de valor (Kassai \& Carvalho, 2016).

Os princípios básicos que sustentam a preparação do IR e disciplinam o conteúdo e a forma de apresentação incluem foco estratégico e perspectiva, conectividade de informações, relacionamento com stakeholders, materialidade, confiabilidade e completude, coerência e comparabilidade (Tribunal de contas da União - TCU, 2019). Os elementos de conteúdo tratam da organização e o ambiente externo, modelo de negócios, riscos e oportunidades, estratégias e alocação de recursos, desempenho, perspectivas, bases para preparação, apresentação e orientação geral (Kassai \& Carvalho, 2016).

O ponto crucial consiste no significado atribuído à palavra "valor"; possíveis interpretações alternativas são "valor para a sociedade" (que é consistente com a contabilidade social e ambiental), "valor para as partes interessadas" (que é consistente com a teoria das partes interessadas da empresa) e "valor para as gerações presentes e futuras" (que é consistente com a sustentabilidade) (Flower, 2015). Para Thompson (2015), o IR pode silenciar os elementos radicais da sustentabilidade e potencialmente reformular práticas corporativas insustentáveis como sustentáveis.

Para Brown e Dillard (2014), no IR questões distributivas foram enquadradas principalmente em termos das perspectivas de custos/benefícios acumulados à empresa e a seus acionistas. Pouca atenção foi dada às distribuições de riqueza e risco para outros, por exemplo, níveis salariais, pagamentos de impostos e todas as classes sociais (Brown \& Dillard, 2014). Por meio de processos internos aprimorados, o IR pode ajudar as organizações a identificar ganhos de eficiência ecológica, como custos de energia reduzidos ou processos de produção mais limpos (Brown \& Dillard, 2014).

Para Zappettini e Unerman (2016), os discursos de sustentabilidade foram "misturados", "incorporados" e "inclinados" a proposições financeiras e macroeconômicas para legitimar a organização no mercado. Para os autores, os processos retóricos envolvidos na construção do IR estavam apoiadas pelo uso de topos de crescimento, desempenho e valor, que se encaixam fortemente em discursos semelhantes introduzidos e promovidos pelo IIRC. A reformulação dos significados de sustentabilidade no IR permitiu uma ressemiotização dos discursos de sustentabilidade, que tende a validar lógicas financeiras e econômicas e a fechar temas ambientais e sociais (Zappettini \& Unerman, 2016).

Gibassier et al. (2018) investigaram o processo pelo qual uma empresa piloto do IIRC adotou o IR, uma inovação gerencial que mescla relatórios financeiros e não financeiros. Para os autores, os gestores da empresa adotaram a dimensão "mítica" do IR residindo nas características aspiracionais do framework. Os resultados encontrados revelaram que os atores organizacionais refletiram sobre a natureza do IR 
e desenvolveram conceituações coletivas e reconceituações do processo de IR. A empresa estudada desafiou a visão do IR sugerida pelo IIRC para manter-se fiel à sua conceituação do IR e implementou sua própria versão do IR (Gibassier et al., 2018).

Para Busco et al. (2018), em vez de preencher a sustentabilidade com significado estável, os gestores foram atraídos pelas lacunas deixadas pelas representações contábeis, levando ao desenvolvimento de novas práticas e desdobramentos de significados em espaços discursivos específicos. Para os autores, o IR contribuiu para a sustentabilidade, permitindo que os indivíduos conectem suas diversas experiências e aspirações ao conceito de sustentabilidade. Dessa forma, o IR pode ser utilizado para acionar as aspirações e as intenções dos gestores em um processo contínuo de questionamento sobre o que é sustentabilidade (Busco et al., 2018).

\subsection{Teoria do Discurso de Laclau e Mouffe}

A teoria do discurso de Laclau e Mouffe (1985) promoveu uma compreensão do social por meio do discurso. Assim, o discurso é uma prática social significativa de natureza material (Mendonça, 2007). Para Laclau e Mouffe (1985), o discurso resulta de palavras e ações sendo resultado da articulação entre elementos linguísticos e extra linguísticos (Lopes \& Mendonça, 2015). O discurso envolve a fixação de significado em domínios particulares e a exclusão de outros possíveis significados (Laclau \& Mouffe, 1985).

Laclau e Mouffe (1985) modificam o conceito de hegemonia de Gramsci (1971) para denotar a estruturação de significados por meio de práticas discursivas (Martin, 2002). A hegemonia representa o momento em que um conteúdo particular assume, em um contexto precário e contingente, a função de representar uma plenitude ausente (Lopes \& Mendonça, 2015). Assim, a hegemonia é alcançada com o deslocamento de modos alternativos de argumentação e formas de atividade, marginalizando diferentes discursos e naturalizando o próprio discurso (Tregidga et al., 2018).

A hegemonia é uma prática de articulação discursiva, em que a articulação é definida como qualquer prática que estabeleça relações entre elementos, resultando na modificação da identidade (Laclau \& Mouffe, 1985). A prática articulatória, por sua vez, consiste na articulação de elementos em um sistema discursivo em torno de um ponto nodal, que fixa parcialmente os sentidos desse sistema (Laclau \& Mouffe, 1985). Essa fixação de significados é necessariamente parcial, tendo em vista as constantes suturas que o discurso sofre na relação com outros discursos concorrentes (Mendonça, 2007).

A partir de um discurso centralizador, um ponto nodal consegue fixar sua significação e, a partir disso, articular elementos que previamente não estavam articulados entre si (Mendonça, 2007). Os pontos nodais são significantes privilegiados em um discurso que une um sistema particular de significado ou cadeia de significação (Howarth \& Stavrakakis, 2000). Tal fixação é sempre parcial, precária e contingente, ou seja, nada pode garantir que determinado discurso consiga articular outros discursos (Mendonça, 2007).

Howarth \& Stavrakakis (2000) observaram que a articulação de um discurso só pode ocorrer em torno de um significante vazio que funciona como um ponto nodal sendo que o vazio é uma qualidade essencial desse ponto. Um significante vazio é, no sentido estrito, um significante sem significado, e permite uma multiplicidade de articulações com significados sem que nenhum deles se estabilize como sentido unívoco (Rodrigues \& Mendonça, 2008).

A possibilidade de ação de qualquer identidade deve ser entendida em sentido relacional: uma identidade busca impor suas vontades na concorrência de outras, visando, universalizar seus conte- 
údos particulares (Mendonça, 2007). Para Laclau (1996), o universal emerge do particular, não como um princípio subjacente que explica o particular, mas como um horizonte incompleto que sutura uma identidade particular deslocada. A hegemonia ocorre somente se a dicotomia universalidade/ particularidade estiver suspensa; sendo que a universalidade existe representando e subvertendo algum objetivo particular e nenhuma particularidade pode existir sem se tornar o locus de efeitos universalizantes (Butler, Laclau \& Zizek, 2000).

\section{METODOLOGIA DA PESQUISA}

Esta pesquisa bibliográfica teve caráter exploratório e descritivo, estruturada com a finalidade de identificar e avaliar os resultados de estudos sobre a temática de interesse. A revisão de literatura foi desenvolvida por meio dos recursos avançados de busca da base de dados Scopus. A triagem dos artigos satisfez a critérios específicos de pesquisa para encontrar a literatura-alvo: i) Os termos "integrated report*"e ("state-owned" ou"public sector") no título, resumo ou palavras-chave; ii) Não houve restrição de data; iii) Lançado como artigos ou "review".

A população deste estudo foi levantada no mês de outubro de 2019 e, posteriormente, atualizada em março de 2020. A base de dados da Scopus identificou 21 publicações que atenderam os critérios de busca. Após a leitura do resumo, foram excluídas três pesquisas que não tratavam sobre o IR, constituindo-se a amostra final em 18 artigos. Ressalta-se que não foram localizadas produções nacionais sobre o tema. Os artigos foram classificados conforme seus aspectos metodológicos e índice de citação do Google Scholar. Para os índices de citação utilizou-se do software Publish or Perish 7.

Para a análise discursiva dos estudos empíricos revisados, utilizou-se da Teoria do Discurso de Laclau e Mouffe (1985). O processo da análise do discurso dos artigos revisados conduziu-se em três etapas: na primeira ocorreu várias leituras dos artigos, e realizada uma redução do volume do texto com a finalidade de construir um conjunto de dados focados na temática da sustentabilidade. O software NVIVO auxiliou na codificação das seções e registro dos extratos relacionados à área de interesse. Os termos utilizados na busca foram: Sustentabilidade, sustentável, sustentáveis e desenvolvimento sustentável. Nesta fase foram excluídos os artigos que não faziam menção a sustentabilidade (Dabbicco, 2015; Macnab, 2015; Veltri \& Silvestri, 2015).

A segunda etapa envolveu a análise dos extratos recuperados em torno do ponto nodal sustentabilidade dos artigos teóricos. Os estudos teóricos foram interpretados com relação a seu posicionamento em relação a sustentabilidade corporativa nos IR do setor público. Como ponto nodal, a sustentabilidade une e sedimenta vários discursos e a forma como ela é constituída consiste em uma tentativa de dominar o campo da discursividade (Laclau \& Mouffe, 1985). Os artigos analisados nesta fase foram: (Cohen \& Karatzimas, 2015; Biondi \& Bracci, 2018; Montesinos \& Brusca, 2019).

Na terceira etapa, foi realizada uma análise da construção do discurso de sustentabilidade nos resultados das pesquisas empíricas sobre os IRs de empresas do setor público. O discurso de sustentabilidade empresarial presente nos artigos empíricos (qualitativos e quantitativos) revisados foram interpretados e categorizados, conforme proposto por Tregidga et al. (2018). A Tabela 1 mostra as categorias de análise do discurso de sustentabilidade dos artigos empíricos. 
Tabela 1 - Categorias de análise do discurso de sustentabilidade

\begin{tabular}{|c|c|}
\hline Categorias & Exemplo \\
\hline Construção/explicação & Triple bottom line com a priorização da dimensão econômica. \\
\hline $\begin{array}{c}\text { Extratos ilustrativos de relatórios } \\
\text { analisados em publicações }\end{array}$ & $\begin{array}{c}\text { "Na Stora Enso acreditamos que o sucesso financeiro a longo prazo é o elemento-chave da } \\
\text { sustentabilidade para os negócios. Excelente desempenho socioambiental não prolongará a vida de } \\
\text { uma empresa economicamente insustentável" (Stora Enso, Relatório de Sustentabilidade, 2005, p. 4 } \\
\text { apud Laine, 2010, p. 265). }\end{array}$ \\
\hline Efeito potencial & $\begin{array}{c}\text { Destaca questões econômicas de progresso, desenvolvimento e crescimento (Laine, 2005; Makela \& } \\
\text { Laine, 2011). Racionalização do lucro (Laine, 2005, 2010; Livesey, 2002a; Milne et al., 2009). }\end{array}$ \\
\hline Pressupostos incorporados & Desenvolvimento sustentável requer rentabilidade corporativa. \\
\hline
\end{tabular}

Fonte: Dados extraídos de Tregidga et al. (2018).

A definição de sustentabilidade do Relatório Brundtland (WCED, 1987) constitui, nos termos de Laclau e Mouffe (1985), uma prática articulatória. A definição articula o desenvolvimento sustentável de maneira que a identidade do conceito seja modificada como resultado da articulação (Tregidga et al., 2018). No entanto, embora parcialmente fixe o significado, continua a ser uma representação universal que une uma série de informações, mas não possui nenhum conteúdo necessário (Laclau, 1992).

É importante destacar que os estudos revisados estão sujeitos a múltiplas camadas de interpretação e construção social. O pesquisador e o pesquisado estão intimamente entrelaçados. Assim, ao reconhecer como o conceito de sustentabilidade nos IRs foi parcialmente preenchido com conteúdo e seus efeitos, tanto os atores dos negócios, quanto os acadêmicos estão envolvidos neste processo de representação da sustentabilidade. Ressalta-se que a análise dos artigos pesquisados foi revisada pelos autores em todo o processo.

\section{APRESENTAÇÃO E DISCUSSÃO DOS RESULTADOS}

Os estudos sobre o IR no setor público ainda estão em fase embrionária (Dumay, Bernardi, Guthrie \& Demartini, 2016). O primeiro artigo filtrado, com base na metodologia adotada, é de 2011, e titulado como"Public sector accountability for cultural assets: An integrated reporting approach", de Wild (2011), um ano após a criação do IIRC. Os estudos mais recentes são Marasca, Montanini, D'Andrea e Cerioni (2020) e Nicolo, Zanellato, Manes-Rossi e Tiron-Tudor (2020). A Tabela 2 apresenta as características dos estudos publicados no período de 2010 a março de 2020.

Os reflexos da quantidade de artigos publicados podem ser justificados com base na evolução do processo de implantação do IR conduzido pelo IIRC, desde sua criação em 2010. De 2010 a 2015, as publicações têm abordagem predominante qualitativa e teórica. Os artigos empíricos publicados, neste período, se referem ao contexto da Africa do Sul. De fato, a África do Sul foi o primeiro país a propor as diretrizes de IR em 2009, emitindo o "King Report on Corporate Governance", conhecido como King III (Institute of Directors Southern Africa - IoDSA, 2009). Em 2019, há um crescimento de publicações sobre a temática, que pode ser justificada pelo lançamento do guia de implementação do IR no setor público "Integrated thinking and reporting. Focusing on value creation in the public sector" publicado pelo IIRC e CIPFA (2016), e a criação Rede de Pioneiros do Setor Público pelo IIRC (2016). Mais de 50\% de todas as publicações que atendem aos critérios de pesquisa foram publicadas nos últimos três anos e este aumento de publicações sobre a temática pode ser justificada pelo aumento de empresas do setor público que divulgaram o IR. 
Tabela 2. Lista dos artigos da amostra.

\begin{tabular}{|c|c|c|c|c|c|}
\hline N. & Autor(es) & Título & Abordagem & G.I. & C.A. \\
\hline 1 & Wild (2011) & $\begin{array}{c}\text { Public sector accountability for cultural assets: An integrated } \\
\text { reporting approach }\end{array}$ & Qualitativo empírico & 02 & 0,22 \\
\hline 2 & Samkin (2012) & $\begin{array}{c}\text { Changes in sustainability reporting by an African defence } \\
\text { contractor: A longitudinal analysis }\end{array}$ & Qualitativo empírico & 45 & 5,63 \\
\hline 3 & Macnab (2015) & $\begin{array}{l}\text { Debate: Would outcome costing and integrated reporting link } \\
\text { resources to strategy in the public sector? }\end{array}$ & Qualitativo teórico & 03 & 0,6 \\
\hline 4 & $\begin{array}{l}\text { Cohen \& Karatzimas } \\
\text { (2015) }\end{array}$ & $\begin{array}{l}\text { Tracing the future of reporting in the public sector: introducing } \\
\text { integrated popular reporting }\end{array}$ & Qualitativo teórico & 48 & 9,6 \\
\hline 5 & Dabbicco (2015) & $\begin{array}{l}\text { The boundary of the public sector in national accounts versus } \\
\text { IPSAS }\end{array}$ & Qualitativo teórico & 08 & 1,6 \\
\hline 6 & Veltri \& Silvestri (2015) & $\begin{array}{l}\text { The Free State University integrated reporting: A critical } \\
\text { consideration }\end{array}$ & Qualitativo empírico & 68 & 13,8 \\
\hline 7 & $\begin{array}{l}\text { Guthrie, Manes-Rossi } \\
\quad \& \text { Orelli (2017) }\end{array}$ & $\begin{array}{l}\text { Integrated reporting and integrated thinking in Italian public } \\
\text { sector organizations }\end{array}$ & Qualitativo empírico & 81 & 27 \\
\hline 8 & Biondi \& Bracci (2018) & $\begin{array}{l}\text { Sustainability, popular and integrated reporting in the public } \\
\text { sector: A fad and fashion perspective }\end{array}$ & Qualitativo teórico & 16 & 8 \\
\hline 9 & $\begin{array}{l}\text { Montecalvo, Farneti \& } \\
\text { De Villiers (2018) }\end{array}$ & $\begin{array}{c}\text { The potential of integrated reporting to enhance sustainability } \\
\text { reporting in the public sector }\end{array}$ & Qualitativo empírico & 23 & 11,5 \\
\hline 10 & $\begin{array}{l}\text { Farneti, Casonato, } \\
\text { Montecalvo \& De } \\
\text { Villiers (2019) }\end{array}$ & $\begin{array}{l}\text { The influence of integrated reporting and stakeholder } \\
\text { information needs on the disclosure of social information in a } \\
\text { state-owned enterprise }\end{array}$ & Qualitativo empírico & 07 & 7 \\
\hline 11 & $\begin{array}{l}\text { Argento, Grossi, } \\
\text { Persson \& Vingren } \\
\text { (2019) }\end{array}$ & $\begin{array}{l}\text { Sustainability disclosures of hybrid organizations: Swedish } \\
\text { state-owned enterprises }\end{array}$ & Quantitativo empírico & 02 & 2 \\
\hline 12 & $\begin{array}{l}\text { Nistor, Stefanescu, } \\
\text { Oprisor \& Crisan } \\
\quad(2019)\end{array}$ & $\begin{array}{l}\text { Approaching public sector transparency through an integrated } \\
\text { reporting benchmark }\end{array}$ & Quantitativo empírico & 01 & 1 \\
\hline 13 & $\begin{array}{l}\text { Cavicchi, Oppi } \\
\text { \&Vagnoni (2019) }\end{array}$ & $\begin{array}{l}\text { On the feasibility of integrated reporting in healthcare: a } \\
\text { context analysis starting from a management commentary }\end{array}$ & Qualitativo empírico & 01 & 1 \\
\hline 14 & $\begin{array}{l}\text { Montesinos \& Brusca } \\
\text { (2019) }\end{array}$ & $\begin{array}{l}\text { Non-financial reporting in the public sector: Alternatives, trends } \\
\text { and opportunities }\end{array}$ & Qualitativo teórico & 07 & 1 \\
\hline 15 & $\begin{array}{l}\text { Caruana \& Grech } \\
\text { (2019) }\end{array}$ & $\begin{array}{l}\text { Tweaking public sector reporting with integrated reporting (IR) } \\
\text { concepts }\end{array}$ & Qualitativo empírico & 1 & 1 \\
\hline 16 & $\begin{array}{l}\text { Tirado-Valencia, } \\
\text { Cordobés-Madueño, } \\
\text { Ruiz-Lozano \& De } \\
\text { Vicente-Lama (2019) }\end{array}$ & $\begin{array}{l}\text { Integrated thinking in the reporting of public sector enterprises: } \\
\text { a proposal of contents }\end{array}$ & Quantitativo empírico & 01 & 1 \\
\hline 17 & Nicolo et al. (2020) & $\begin{array}{l}\text { Corporate reporting metamorphosis: empirical findings from } \\
\text { state-owned enterprises }\end{array}$ & Quantitativo empírico & 00 & 0 \\
\hline 18 & Marasca et al. (2020) & $\begin{array}{l}\text { The how and why of integrated reporting in a public health care } \\
\text { organization: The stakeholders' perspective }\end{array}$ & Qualitativo empírico & 00 & 0 \\
\hline
\end{tabular}

Dentre os estudos analisados, destaca-se pelo número de citações o trabalho de Guthrie et al. (2017) que analisaram como mecanismos internos de mudança podem levar as organizações a adotar o IR e a buscar o pensamento integrado. Os autores investigaram cinco organizações do setor público italiano, por meio de documentos oficiais, press releases e entrevistas semiestruturadas com os gestores. Os achados da pesquisa revelaram que o IR está mais próximo da estratégia e da criação de valor, de forma que a adoção ao framework do IR levou ao pensamento integrado nas empresas pesquisadas (Guthrie et al., 2017).

Na sequência, realizou-se uma análise discursiva dos estudos revisados sobre o IR no setor público, em torno do ponto nodal sustentabilidade. Laclau e Mouffe (1985) apontam que, uma maneira de alcançar a hegemonia é definir o espaço discursivo de maneira que o faça substituindo um conceito vazio por outro. Essa substituição é evidenciada no discurso corporativo sobre sustentabilidade nos IR das pesquisas revisadas, em que o significado permanece vazio, mas encapsula muitos outros aspectos da sustentabilidade e racionaliza a primazia da economia.

A sustentabilidade nos IRs evidenciados nas pesquisas acadêmicas analisadas surgem como algo geral, mas basicamente positivo e importante. Consiste em uma ideia que deve ser seguida e parece haver benefícios para a empresa ligada ao setor público adotar e aplicar consistentemente os princípios do IR em suas atividades (Montesinos \& Brusca, 2019; Guthrie et al., 2017; Farneti et al., 2019). Para 
Montesinos e Brusca (2019), o IR pode ser implementado no setor público com mínimas adaptações derivadas das características específicas dessas entidades.

Neste sentido, Biondi e Bracci (2018) alertam que inovações, como o IR, têm muito em comum com os frameworks anteriores e que há o risco de criar apenas novos rótulos, sem inovação real ou melhoria da responsabilidade pública. Além disso, há também o risco de que uma ferramenta de reporte unitária seja tão ampla em alcance e conteúdo que derrota seu próprio propósito de ser fácil de ler e acessível a não especialistas (Biondi \& Bracci, 2018).

Cohen e Karatzimas (2015) se concentram nas necessidades de informação dos cidadãos, um grupo de stakeholders do setor público cujas necessidades de informação parecem ser tratadas em um nível subótimo sob o status quo existente. Os autores propõem os "Relatórios Populares Integrados - IPR" a partir da sintetização de elementos de relatórios populares e do IR.

A Tabela 3 exemplifica as construções do discurso de sustentabilidade nos estudos empíricos pesquisados com base em citações do IR das organizações pesquisadas nestes artigos. Pela análise de cada uma dessas construções e dos resultados obtidos, foram identificados os efeitos potenciais, bem como as premissas embutidas nas construções sobre sustentabilidade. Trechos desta análise discursiva dos artigos empíricos são apresentados na Tabela 3.

Os discursos de sustentabilidade pesquisados podem ser vistos como articulações que contêm duas características aparentemente contraditórias: certeza e imprecisão. As construções do conceito de sustentabilidade analisadas, tem um elemento de certeza. As declarações são apresentadas como verdadeiras e as empresas se posicionam como conhecedoras do que é sustentabilidade, e como ela pode ser alcançada com a prática do negócio. A presença de certeza de que a adoção ao IR amplia a visão de sustentabilidade é essencial ao potencial hegemônico do discurso e à capacidade de fixar parcialmente o significado em torno do ponto nodal.

No entanto, Montecalvo et al. (2018) alertam que no discurso corporativo de sustentabilidade analisado por eles"não abrangem todos os aspectos da sustentabilidade, em especial a equidade intergeracional. A adoção do IR foi, neste caso, em grande parte impulsionada pelo isomorfismo normativo" (Montecalvo et al., 2018, p. 14-15).

Assim, as tentativas das organizações de preencher com significado de sustentabilidade em seus IRs podem ser consideradas como uma tentativa de obter (ou manter) o controle hegemônico do espaço discursivo sobre. Ao mesmo tempo, os resultados revelam que o discurso corporativo da sustentabilidade também é vago. A imprecisão do termo é reconhecida por vários autores (Montecalvo, 2018; Samkin, 2012):

De 2009 a 2011, o aumento do uso do termo pela administração na preparação dos relatórios garantiu um cluster ou tema de "sustentabilidade" em cada período do relatório. No entanto, o estudo constatou que os conceitos que compõem o cluster ou tema "sustentabilidade" mudavam anualmente. Esta constatação não deve ser inesperada, pois é consistente com as dificuldades que a gerência da Denel parecia ter ao relatar problemas de sustentabilidade (Samkin, 2012, p. 160, grifo nosso).

Ainda assim, o conceito de sustentabilidade nos IRs permanece vago (Samkim, 2012; Biondi \& Bracci, 2018; Montecalvo et al., 2018). No discurso corporativo em torno do ponto nodal sustentabilidade, são elaboradas referências a sustentar a economia, o meio ambiente e a sociedade. No entanto, o que essas dimensões realmente consistem, não está claro, e se representam apenas uma referência à sustentação do desempenho financeiro da organização. 
Os resultados dessa pesquisa revelam que, ao fechar a sustentabilidade em torno de enquadramentos de business-case, conforme evidenciado na Tabela 3, os IRs ignoram e obscurecem a possiblidade de (re)imaginar os IRs por caminhos mais pluralistas (Brown \& Dillard, 2014). Para Argento et al. (2019) devido à natureza híbrida das empresas estatais (SOEs), eles devem comunicar informações de sustentabilidade a diversos stakeholders com diferentes interesses. No entanto, os resultados de Argento et al. (2019) revelam que:

Primeiro, a propriedade do Estado afeta negativamente o relatório de sustentabilidade dos SOEs; em outras palavras, como a porcentagem de propriedade do Estado aumenta, a divulgação de informações de sustentabilidade diminui. Em segundo lugar, o tamanho de um SOE tem uma relação positiva com suas divulgações de sustentabilidade (Argento et al., 2019, p. 524, grifo nosso).

Caruana e Grech (2019) não recomendam a introdução completa dos requisitos de IR no setor público, pois as consequências podem ser avassaladoras e dispendiosas. Para eles, seria mais sensato conhecer o framework do IR e extrair dele apenas o que é considerado relevante. Os autores destacam que o IR foi criticado por se concentrar nas necessidades dos investidores e não na responsabilidade e na sustentabilidade (Caruana \& Grech, 2019).

Tirado-Valencia et al. (2019, p. 14) relatam que:

[...] os processos internos devem ser melhor coordenados e que há necessidade de maior envolvimento dos gestores com aspectos não financeiros. Além disso, as informações devem ser mais interconectadas. Isso melhoraria a percepção que os cidadãos têm da sustentabilidade futura dos entes públicos.

Tabela 3. Categorias de análise do discurso de sustentabilidade das pesquisas empíricas revisadas

\begin{tabular}{|c|c|c|c|}
\hline Construção/explicação & $\begin{array}{l}\text { Extratos dos IRs analisados pelas pesquisas } \\
\text { acadêmicas }\end{array}$ & Efeito potencial & Suposições incorporadas \\
\hline $\begin{array}{l}\text { Sustentabilidade com } \\
\text { priorização da questão } \\
\text { econômica }\end{array}$ & $\begin{array}{l}\text { "isso não representa uma atividade central do } \\
\text { hospital e há algumas dificuldades em traduzir a } \\
\text { sustentabilidade em termos operacionais" (AD-LHA } \\
\text { in Cavichi et al., 2019, 361). }\end{array}$ & $\begin{array}{l}\text { A sustentabilidade é } \\
\text { relacionada principalmente a } \\
\text { metas financeiras, em vez de } \\
\text { metas sociais e ambientais. }\end{array}$ & $\begin{array}{l}\text { Sustentabilidade não é uma } \\
\text { questão relevante }\end{array}$ \\
\hline $\begin{array}{l}\text { Sustentabilidade como } \\
\text { sinônimo de adoção ao IR }\end{array}$ & $\begin{array}{l}\text { "Queríamos construir além do GRI... Havia.... } \\
\text { interesse em... contar uma história mais ampla, } \\
\text { porque temos [muitas] partes interessadas e nós... } \\
\text { foram [não] atendê-los no nosso melhor. Então [IR] } \\
\text { parecia atraente. Fomos convidados a participar... } \\
\text { o Programa Piloto, e foi aí que começou" (AA in } \\
\text { Montecalvo et al., 2018, p. 12). }\end{array}$ & $\begin{array}{l}\text { O IR está se tornando } \\
\text { uma norma aceita } \\
\text { para os relatórios de } \\
\text { sustentabilidade }\end{array}$ & $\begin{array}{l}\text { O framework do IR abrange } \\
\text { questões de sustentabilidade. }\end{array}$ \\
\hline $\begin{array}{l}\text { Sustentabilidade } \\
\text { integrada a estratégia } \\
\text { empresarial }\end{array}$ & $\begin{array}{l}\text { "Decidimos impulsionar a sustentabilidade dentro } \\
\text { de nossa organização. O relatório integrado exige... } \\
\text { uma opinião sobre sustentabilidade, estratégia, } \\
\text { materialidade e o uso de novas ferramentas e } \\
\text { métricas para levar em conta todos esses aspectos. } \\
\text { É a maneira mais rápida de definir sustentabilidade" } \\
\text { (Gerente de Sustentabilidade A in Guthrie et al., } \\
\text { 2017, p. 12). }\end{array}$ & $\begin{array}{c}\text { A adoção ao IR pode } \\
\text { impulsionar a mudança } \\
\text { organizacional para } \\
\text { integrar a sustentabilidade } \\
\text { à atividade principal da } \\
\text { organização }\end{array}$ & $\begin{array}{l}\text { Sustentabilidade é compatível } \\
\text { com o "business-case" }\end{array}$ \\
\hline $\begin{array}{l}\text { Sustentabilidade com } \\
\text { priorização da dimensão } \\
\text { econômica }\end{array}$ & $\begin{array}{l}\text { Procurar constantemente reduzir o impacto } \\
\text { ambiental das operações da NZ Post, quando isso } \\
\text { for comercialmente viável. Redução de emissão } \\
\text { de carbono, eficiência energética e redução de } \\
\text { resíduos e reciclagem são áreas-chave para esta } \\
\text { empresa, e continuaremos a estabelecer metas } \\
\text { específicas no negócio, com fornecedores e clientes } \\
\text { (Relatório Anual, 2017, p. } 34 \text { in Farnetti et al., 2019, } \\
\text { p. 14). }\end{array}$ & $\begin{array}{l}\text { A empresa vincula } \\
\text { claramente questões } \\
\text { de sustentabilidade a } \\
\text { viabilidade financeira. }\end{array}$ & $\begin{array}{l}\text { O IR considera a criação de valor } \\
\text { ao investidor em primeiro lugar. } \\
\text { Demais stakeholders ficam em } \\
\text { segundo lugar. }\end{array}$ \\
\hline $\begin{array}{l}\text { Sustentabilidade como um } \\
\text { conceito indefinido }\end{array}$ & $\begin{array}{l}\text { "o monitoramento e a comunicação de questões } \\
\text { de sustentabilidade é uma disciplina em evolução } \\
\text { dentro do grupo, e estão sendo tomadas ações } \\
\text { para formalizar a estratégia, estruturas de } \\
\text { governança, políticas e processos de comunicação } \\
\text { da empresa" (Denel (Pty) Limited, 2009, p. } 61 \text { in } \\
\text { Samkin, 2012, p. 160). }\end{array}$ & $\begin{array}{l}\text { Dificuldade dos gestores } \\
\text { em relatar questões de } \\
\text { sustentabilidade. }\end{array}$ & $\begin{array}{l}\text { Dependendo da ênfase } \\
\text { da empresa, o conceito de } \\
\text { sustentabilidade pode ser } \\
\text { definido de formas diferentes. }\end{array}$ \\
\hline
\end{tabular}


A construção da sustentabilidade no contexto corporativo, e em especial no IR no setor público, é resultado de um processo político no qual os aspectos da sustentabilidade não são apenas marginalizados e excluídos, mas também são ativamente promovidos no discurso organizacional. No entanto, é a constatação de que existem alternativas, antagonismos e outras possibilidades, que mantêm vivo o potencial de combater tentativas de fechamento discursivo e hegemonia (Tregidga et al., 2018). Neste sentido, existe a necessidade de pesquisas acadêmicas com o intuito de debater o mérito do IR no setor público como uma iniciativa de mudança que contribui para a sustentabilidade.

\section{CONSIDERAÇÕES FINAIS}

Analisou-se, neste artigo, a construção do discurso de sustentabilidade nos resultados de pesquisas sobre os IRs de empresas do setor público. No geral, os discursos de sustentabilidade presentes nos estudos revisados foram incorporados a proposições financeiras para se adequar a narrativas organizacionais e validar as atividades das organizações e retratar a organização como um agente confiável aos olhos do público externo e enfatizar a criação de valor, conforme preconizado por Zappettini e Unerman (2016) e Tregidga et al. (2018). No entanto, em nível gerencial destacou-se o potencial libertador e emancipatório do IR no setor público, conforme constatado por Guthrie et al. (2017), Montesinos e Brusca (2019) e Farneti et al., (2019).

É importante salientar que não há soluções simples para os desafios da avaliação e do engajamento da sustentabilidade, principalmente por causa de sua natureza política e carregada de valor (Brown \& Dillard, 2014). Existe a necessidade de engajamento acadêmico na investigação sobre aspectos de sustentabilidade nos IR, particularmente em relação ao discurso hegemônico no contexto corporativo. A sustentabilidade é um discurso político, e os acadêmicos são, juntamente com outros, atores políticos de forma a desempenhar um importante papel na construção de um discurso externo (Tregidga et al., 2018).

Para futuras pesquisas sobre o discurso de sustentabilidade nos IRs, existe a necessidade de: i) "vigilância e conscientização" (Tregidga et al., 2018, p. 317) ao conduzir estudos empíricos sobre o IR e as ações e desempenho relacionados a sustentabilidade; ii) "análises críticas e reflexivas" (Tregidga et al., 2018, p. 317) dos IR, em especial no contexto social e ecológico mais amplo em que operam; iii) "ampliar e abrir" (Brown \& Dillard, 2014, p. 1120) o conceito de sustentabilidade inserido no framework do IR, ao fornecer alternativas/adaptações de relatos.

Em primeiro lugar, é preciso que a pesquisa envolvida na crítica à sustentabilidade nos IRs em empresas do setor público entendam sua natureza discursiva e permaneçam vigilante com seu caráter político. Esta percepção está presente em trabalhos recentes do discurso de sustentabilidade nos IRs (Zappettini \& Unerman, 2016; Busco et al., 2018).

Em segundo lugar, existe a necessidade de análises críticas e reflexivas, por exemplo, há oportunidades de pesquisas acadêmicas criarem discursos alternativos ou legitimarem o discurso produzido pelas empresas no IR. Um corpo crescente desses últimos, oriundo da literatura contábil e de relatórios, tais como, Brown \& Dillard (2014), Flower (2015) e Thompson (2015). Análises críticas e reflexivas também poderiam ser realizadas por meio da colaboração com outras áreas fora da contabilidade.

Ademais, para que a pesquisa acadêmicas se mostre eficaz, ela deve ir além da vigilância, conscientização e crítica contextual e retórica: ela também deve articular novas alternativas, novos quadros de referência para imaginar novas maneiras de pensar e decidir e, em alguns casos, isso pode significar retornar a ideias anteriormente expressas, mas negligenciadas. 
Futuras pesquisas engajadas podem buscar entender o discurso de sustentabilidade, ao abordar: Como o IR como prática inovadora pode contribuir para a sustentabilidade? Como outros pontos nodais do discurso podem ser usados ao formular seus discursos de sustentabilidade nos IRs? Pesquisas normativas devem examinar mecanismos alternativos de prestação de contas e governança e/ou propor adaptações do IR no setor público.

Além disso, são necessárias pesquisas futuras para compreender os espaços discursivos da sustentabilidade em diferentes níveis, em particular, nos espaços oferecidos por projetos específicos na implantação do IR no setor público. Verifica-se que, ainda há pouca evidência empírica das consequências do IR no discurso de sustentabilidade corporativo. Essas são as áreas em que mais pesquisas são necessárias, e que se envolvam criticamente e ofereçam alternativas e possíveis soluções.

Uma melhor compreensão e apreciação das complexidades, dificuldades e sensibilidades dos IRs no setor público possibilitarão que acadêmicos e profissionais desenvolvam essa prática de maneira a apoiar um futuro econômico, ecológico e social sustentável. As contribuições deste estudo consistem na metodologia que se aplica a aspectos importantes da pesquisa sobre o discurso de sustentabilidade no IR do setor público no que tange às reflexões sobre como a pesquisa sobre a temática pode avançar. As principais lacunas ainda permanecem, uma vez que as pesquisas ainda estão em fase inicial de desenvolvimento.

Este estudo possui limitações relacionadas ao método de pesquisa aplicado, em particular, no que diz respeito ao processo de busca e escolha dos filtros para se chegar à amostra de artigos analisados, uma vez que se excluir pesquisas importantes nessa área do conhecimento. A amostra foi restrita à base de dados Scopus, nas quais pesquisas brasileiras com a temática não foram encontradas na referida base de dados no portal da Capes. Além disso, a análise teve como base os resultados evidenciados pelos artigos revisados em torno do ponto nodal sustentabilidade. Outros pesquisadores, usando os mesmos dados, poderão apresentar diferentes interpretações e conclusões.

\section{REFERÊNCIAS}

Argento, D., Grossi, G., Persson, K., \& Vingren, T. (2019). Sustainability disclosures of hybrid organizations: Swedish state-owned enterprises. Meditari Accountancy Research, 27(4), 505-533. http://doi. org/10.1108/MEDAR-07-2018-0362

Atkins, J., Atkins, B. C., Thomson, I., \& Maroun, W. (2015). “Good” news from nowhere: Imagining utopian sustainable accounting. Accounting, Auditing and Accountability Journal, 28(5), 651-670. http://doi. org/10.1108/AAAJ-09-2013-1485

Bebbington, J., \& Gray, R. (2001). An account of sustainability: failure, success and a reconceptualization. Critical perspectives on accounting, 12(5), 557-587. https://doi.org/10.1006/cpac.2000.0450

Biondi, L., \& Bracci, E. (2018). Sustainability, popular and integrated reporting in the public sector: A fad and fashion perspective. Sustainability (Switzerland), 10(9), 1-16. http://doi.org/10.3390/su10093112

Brown, J., \& Dillard, J. (2014). Integrated reporting: On the need for broadening out and opening up. Accounting, Auditing and Accountability Journal, 27(7), 1120-1156. http://doi.org/10.1108/AAAJ-042013-1313

Busco, C., Frigo, M. L., Paolo, Q., \& Angelo, R. (2013). Redefining corporate accountability through integrated reporting: What happens when values and value creation meet? Strategic Finance, August, 
33-41. https://sfmagazine.com/wp-content/uploads/sfarchive/2013/08/Redefining-Corporate-Accountability-through-Integrated-Reporting.pdf

Busco, C., Giovannoni, E., Granà, F., \& Izzo, M. F. (2018). Making sustainability meaningful: aspirations, discourses and reporting practices. Accounting, Auditing and Accountability Journal, 31(8), 2218-2246. http://doi.org/10.1108/AAAJ-04-2017-2917

Butler, J., Laclau, E., Žižek, S., \& Zizek, S. (2000). Contingency, hegemony, universality: Contemporary dialogues on the left. Verso.

Caruana, J., \& Grech, I. (2019). Tweaking public sector reporting with integrated reporting (IR) concepts. Public Money \& Management, 39(6), 409-417. http://doi.org/10.1080/09540962.2019.1583911

Cavicchi, C., Oppi, C., \& Vagnoni, E. (2019). On the feasibility of integrated reporting in healthcare: a context analysis starting from a management commentary. Journal of Management and Governance, 23(2), 345-371. http://doi.org/10.1007/s10997-019-09456-2

Cohen, S., \& Karatzimas, S. (2015). Tracing the future of reporting in the public sector: introducing integrated popular reporting. International Journal of Public Sector Management, 28(6), 449-460. http:// doi.org/10.1108/JJPSM-11-2014-0140

Dabbicco, G. (2015). The impact of accrual-based public accounting harmonization on EU macroeconomic surveillance and governments' policy decision-making. International Journal of Public Administration, 38(4), 253-267. http://doi.org/10.1080/01900692.2015.999581

Drexhage, J., \& Murphy, D. (2010). Sustainable development: from Brundtland to Rio 2012. United Nations Headquarters, New York, 2010, 9-13. https://www.e-education.psu.edu/emsc302/sites/www.e-education.psu.edu.emsc302/files/Sustainable\%20Development_from\%20Brundtland\%20to\%20Rio\%20 2012\%20\%281\%29.pdf

Dumay, J., Bernardi, C., Guthrie, J., \& Demartini, P. (2016). Integrated reporting: a structured literature review. Accounting Forum, 40(3), 166-185. http://doi.org/0.1016/j.accfor.2016.06.001

Dumay, J., Guthrie, J., \& Farneti, F. (2010). GRI sustainability reporting guidelines for public and third sector organizations: A critical review. Public Management Review, 12(4), 531-548. http://doi.org/10.1 080/14719037.2010.496266

Elkington, J. (1997). Cannibals with Forks: the TBL of the 21st Century Business. Oxford: Capstone Publishing.

Farneti, F., Casonato, F., Montecalvo, M., \& De Villiers, C. J. (2019). The influence of integrated reporting and stakeholder information needs on the disclosure of social information in a state-owned enterprise. Meditari Accountancy Research, 27, 556-579. http://doi.org/10.1108/MEDAR-01-2019-0436

Figueira, I., Domingues, A. R., Caeiro, S., Painho, M., Antunes, P., Santos, R., ... \& Ramos, T. B. (2018). Sustainability policies and practices in public sector organisations: The case of the Portuguese Central Public Administration. Journal of Cleaner Production, 202, 616-630. http://doi.org/10.1016/j.jclepro.2018.07.244

Flower, J. (2015). The international integrated reporting council: a story of failure. Critical Perspectives on Accounting, 27, 1-17. http://doi.org/10.1016/j.cpa.2014.07.002 
García-Sánchez, I. M., Frías-Aceituno, J.V., \& Rodríguez-Domínguez, L. (2013). Determinants of corporate social disclosure in Spanish local governments. Journal of Cleaner Production, 39, 60-72. http://doi. org/10.1016/j.jclepro.2012.08.037

Gibassier, D., Rodrigue, M., \& Arjaliès, D. L. (2018). Integrated reporting is like God: no one has met Him, but everybody talks about Him. Accounting, Auditing \& Accountability Journal, 31(5), 1349-1380. http:// doi.org/10.1108/AAAJ-07-2016-2631

Gramsci, A. (1971). Selections from the prison notebooks of Antonio Gramsci. Lawrence \& Wishart.

Granà, F. (2018). Representing sustainability within organizations: the role of accounting and reporting practices. [Doctoral's thesis in Philosophy, National University of Ireland. J.E. Cairnes School of Business \& Economics]. https://aran.library.nuigalway.ie/bitstream/handle/10379/14611/PhD\%20 Thesis\%20-\%20Fabrizio\%20Gran\%C3\%A0\%20-\%20Representing\%20sustainability\%20within\%20 organizations_the\%20role\%20of\%20accounting\%20and\%20reporting\%20practices\%20.pdf?sequen$\mathrm{ce}=1$ \&isAllowed $=\mathrm{y}$

Gray, R. (2010). Is accounting for sustainability actually accounting for sustainability ... and how would we know? An exploration of narratives of organisations and the planet. Accounting, organizations and society, 35(1), 47-62. http://doi.org/10.1016/j.aos.2009.04.006

Guthrie, J., Manes-Rossi, F., \& Orelli, R. L. (2017). Integrated reporting and integrated thinking in Italian public sector organisations. Meditari Accountancy Research, 25(4), 553-573. http://doi.org/10.1108/ MEDAR-06-2017-0155

Howarth, D., \& Stavrakakis, Y. (2000). Introducing discourse theory and political analysis. In: D., Howarth; A., Norval, \& Y., Stavrakakis. Discourse theory and political analysis: Identities, hegemonies, and social change. Manchester University Press.

Humphrey, C., O'Dwyer, B., \& Unerman, J. (2017). Re-theorizing the configuration of organizational fields: the IIRC and the pursuit of 'Enlightened' corporate reporting. Accounting and Business Research, 47(1), 30-63. http://doi.org/10.1080/00014788.2016.1198683

Institute of Directors Southern Africa (IoDSA). (2009). King Report on Governance for South Africa, and the King Code of Governance Principles. https://cdn.ymaws.com/www.iodsa.co.za/resource/resmgr/ king_iii/King_Report_on_Governance_fo.pdf

International Integrated Reporting Council (IIRC) \& Chartered Institute of Public Finance and Accounting (CIPFA). (2016), Focusing on value creation in the public sector. Copyright $\odot$ September 2016 by the International

Integrated Reporting Council ('the IIRC'). Used with permission of the IIRC. https://integratedreporting. org/wp-content/uploads/2016/09/Focusing-on-value-creation-in-the-public-sector-_vFINAL.pdf

International Integrated Reporting Council (IIRC) (2016). Introducing the Public Sector Pioneer Network. https://integratedreporting.org/wp-content/uploads/2014/07/Public-Sector-Pioneer-Network-flyer.pdf

porting Council (IIRC). (2013). A estrutura internacional para Relato Integrado. Recuperado de http:// integratedreporting.org/wp-content/uploads/2015/03/13-12-08-THE-INTERNATIONAL-IR-FRAMEWORK-Portugese-final-1.pdf 
Kassai, J. R., \& Carvalho, N. (2016). Intangible assets market value: Integrated Reporting: When, Why and How Did It Happen? In Integrated Reporting (p. 109-116). Palgrave Macmillan. http://www.oceantomo. com/2015/03/04/2015-intangible-asset-market-value-study/

Laclau, E. (1992). Universalism, Particularism, and the Question of Identity. October, 61, 83-90. http:// doi.org/10.2307/778788

Laclau, E. (1996). Por qué los significantes vacíos son importantes para la política? in Emancipación y Diferencia, Buenos Aires, Ariel.

Laclau, E., \& Mouffe, C. (1985). Hegemony and state socialism: towards a radical democratic politics. Verso.

Laine, M. (2005). Meanings of the term 'sustainable development'in Finnish corporate disclosures. Accounting Forum 29(4), 395-413. https://doi.org/10.1016/j.accfor.2005.04.001

Laine, M. (2010). Towards sustaining the status quo: Business talk of sustainability in Finnish corporate disclosures 1987-2005. European Accounting Review, 19(2), 247-274. http://doi. org/10.1080/09638180903136258

Lopes, A. C., \& de Mendonça, D. (Eds.). (2015). A teoria do discurso de Ernesto Laclau: ensaios críticos e entrevistas. Annablume.

Macnab, A. (2015). Debate: Would outcome costing and integrated reporting link resources to strategy in the public sector?. Public Money \& Management, 35(6), 399-400. http://doi.org/10.1080/09540962 .2015 .1083683

Makela, H., \& Laine, M. (2011). A CEO with many messages: Comparing the ideological representations provided by different corporate reports. Accounting Forum, 35(4), 217-231. http://doi.org/10.1016/j. accfor.2011.06.008.

Marasca, S., Montanini, L., D'Andrea, A., \& Cerioni, E. (2020). The how and why of integrated reporting in a public health care organization:The stakeholders' perspective. Business Strategy and the Environment, 29(4), 1714-1722. http://doi.org/10.1002/bse.2463

Martin, J. (2002). The political logic of discourse: a neo-Gramscian view. History of European Ideas, 28(12), 21-31. http://doi.org/10.1016/S0191-6599(02)00004-9

Mendonça, D. (2007). A teoria da hegemonia de Ernesto Laclau e a análise política brasileira. Ciências Sociais Unisinos, 43(3), 249-258. http://revistas.unisinos.br/index.php/ciencias_sociais/article/view/5674

Milne, M. J., and Gray, R. (2013). W(h)ither ecology? The triple bottom line, the global reporting initiative, and corporate sustainability reporting. Journal of business ethics, 118(1), 13-29. http://doi.org/10.1007/ s10551-012-1543-8

Milne, M. J., Kearins, K., and Walton, S. (2006). Creating adventures in wonderland:Thejourney metaphor and environmental sustainability. Organization, 13(6), 801-839. http://doi.org/10.1177/1350508406068506

Montecalvo, M., Farneti, F., \& De Villiers, C. (2018). The potential of integrated reporting to enhance sustainability reporting in the public sector. Public Money \& Management, 38(5), 365-374. http://doi. org/10.1080/09540962.2018.1477675

Montesinos, V., \& Brusca, I. (2019). Non-financial reporting in the public sector: alternatives, trends and opportunities. Revista de Contabilidad-Spanish Accounting Review, 22(2), 122-128. http://doi. org/10.6018/rcsar.383071 
Nicolo, G., Zanellato, G., Manes-Rossi, F., \& Tiron-Tudor, A. (2020). http://doi.org/10.1080/09540962.20 20.1719633

Nistor, C. S., Stefanescu, C. A., Oprisor, T., \& Crisan, A. R. (2019). Approaching public sector transparency through an integrated reporting benchmark. Journal of Financial Reporting and Accounting, 17(2), 249-270. http://doi.org/10.1080/09540962.2020.1719633

Rodrigues, L. P., and Mendonça, D. 2008. Pós-estruturalismo e teoria do discurso: em torno de Ernesto Laclau. EdiPUCRS.

Samkin, G. (2012). Changes in sustainability reporting by an African defence contractor: a longitudinal analysis. Meditari Accountancy Research, 20(2), 134-166. http://doi.org/10.1108/10222521211277834

Silva, K., Yapa, P. W. S., \& Vesty, G. (2020). The Impact of Accountability Mechanisms on Public Sector Environmental Sustainability Performance: A Case Study of Sri Lanka. Australasian Accounting, Business and Finance Journal, 14(3), 38-55. http://doi.org/10.14453/aabfj.v14i3.4

Stiglitz, J. E., Sen, A., \& Fitoussi, J. P. (2009). Report by the commission on the measurement of economic performance and social progress. http://www.stiglitz-sen-fitoussi.fr/en/documents.htm

Thompson, I. (2015). "But does sustainability need capitalism or an integrated report" a commentary on "The International Integrated Reporting Council: A story of failure" by Flower, J. Critical Perspectives on Accounting, 27,18-22. http://doi.org/10.1016/j.cpa.2014.07.003

Tirado-Valencia, P., Cordobés-Madueño, M., Ruiz-Lozano, M. and De Vicente-Lama, M. (2019). Integrated thinking in the reporting of public sector enterprises: a proposal of contents. Meditari Accountancy Research, 28(3), 435-453. https://doi.org/10.1108/MEDAR-03-2019-0458

Tregidga, H. (2007). Power and politics of organisational sustainable development: an analysis of organisational reporting discourse [Doctoral dissertation, University of Otago, New Zealand.

Tregidga, H., Milne, M. J., \& Kearins, K. (2018). Ramping up resistance: Corporate sustainable development and academic research. Business \& Society, 57(2), 292-334. http://doi.org/10.1177/0007650315611459

Tribunal de Contas da União (TCU) (2019). Relatório de gestão: Guia para elaboração na forma de relatório integrado, [2a.ed.] https://portal.tcu.gov.br/biblioteca-digital/relatorio-de-gestao-guia-para-elaboraca o-na-forma-de-relatorio-integrado-segunda-edicao-A81881E6E79824C016E8FD665 1B7F94.htm

Veltri, S., \& Silvestri, A. (2015). The Free State University integrated reporting: a critical consideration. Journal of Intellectual Capital, 16(2), 443-462. http://doi.org/10.1108/JIC-06-2014-0077

Vizeu, F., Meneghetti, F. K., \& Seifert, R. E. (2012). Por uma crítica ao conceito de desenvolvimento sustentável. Cadernos Ebape, 10(3), 569-583. http://doi.org/10.1590/S1679-39512012000300007

Voss, B. L., Carter, D. B., \& Salotti, B. M. (2017). Hegemonies, Politics, and the Brazilian Academy in Social and Environmental Accounting: A Post-Structural Advances in Environmental Accounting \& Management, 6, 13-68. http://doi.org/10.1108/S1479-359820160000006001

Wild, S. (2011). Public sector accountability for cultural assets: An integrated reporting approach. International Journal of Environmental, Cultural, Economic and Social Sustainability, 7(5), 379-390. https:// doi.org/10.18848/1832-2077/CGP/v07i05/54999

Word Commission on Environment and Development-WCED. (1987). Our common future, 17, 1-91. 
Zappettini, F., \& Unerman, J. (2016). 'Mixing' and 'Bending': the recontextualisation of discourses of sustainability in integrated reporting. Discourse and Communication, 10(5), 521-542. http://doi. org/10.1177/1750481316659175 